\title{
ALTA PREVALENCIA DE IGG ANTI CITOMEGALOVIRUS EN 583 EMBARAZOS: HOSPITAL PADRE HURTADO *
}

\author{
Masami Yamamoto C. ${ }^{1}$, Priscilla Prado D. 1, Jan Wilhelm B. ${ }^{1}$, Russel Bradford ${ }^{2}$,

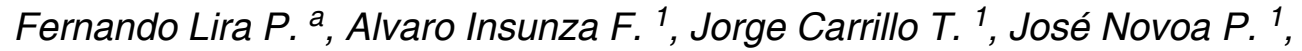 \\ Loretto Vergara M. ${ }^{1}$, Enrique Paiva W. ${ }^{1}$ \\ ${ }^{1}$ Hospital Padre Hurtado, Facultad de Medicina Clínica Alemana, Universidad del Desarrollo. ${ }^{2}$ Departamento de \\ Pediatría, The Alabama University at Birmingham.
}

\footnotetext{
a Alumno, Escuela de Medicina, Pontificia Universidad Católica de Chile.

* Estudio financiado por la American Academy of Pediatrics, Resident's Grant.
}

\section{RESUMEN}

Antecedentes: Citomegalovirus (CMV) es la infección congénita más frecuente, demostrado en el $1 \%$ de recién nacidos en países desarrollados. Es la primera causa de sordera y alteraciones del desarrollo neurológico infantil. Recientes estudios han demostrado que la seropositividad no evita una reinfección materna ni la enfermedad congénita, por lo que la caracterización de la seroprevalencia permite saber si la infección congénita proviene mayoritariamente de primoinfección o de reinfección. Objetivos: Conocer la seroprevalencia al parto en 583 mujeres beneficiarias del Hospital Padre Hurtado durante mayo y junio del 2006. Métodos: Estudio prospectivo, observacional, en que se estudio la presencia de IgG anti CMV en sangre materna al parto. Resultados: Se obtuvo una seroprevalencia de $95 \%$, sin casos de infección sintomática al nacer. Conclusión: La seroprevalencia es elevada, lo que sugiere que la reinfección sería la forma principal de infección congénita. Un estudio en recién nacidos con cultivos virales o PCR permitiría conocer la tasa de infección congénita real, y no un estudio basado en seroconversión pues omitiría todos los casos que reinfección, que serían mayoritarios.

\section{PALABRAS CLAVE: Citomegalovirus congénito, tamizaje, serología, prevalencia}

\section{SUMMARY}

Background: Cytomegalovirus is the most frequent congenital infection, affecting $1 \%$ of the population in developed countries, and the leading cause of deafness and brain development abnormalities in children. Recent studies have demonstrated that seropositivity do not avoid reinfection and congenital disease. Objective: To study the seroprevalence in 583 pregnant women at delivery at Padre Hurtado Hospital, during 2006. Methods: Prospective, observational study, in which maternal blood at delivery was studied for the presence of anti CMV IgG. Results: There was $95 \%$ seroprevalence, without any case of symptomatic infection. Conclusion: The high prevalence supports that most of the cases of congenital disease would occur in seropositive women, supporting that reinfection is the main way of neonatal compromise. This supports that a study with direct detection in liveborns would be suitable to reveal the impact of cytomegalovirus in our population and not that of seroconversion.

KEY WORDS: Congenital cytomegalovirus, screening, serology, prevalence 


\section{INTRODUCCIÓN}

El Citomegalovirus (CMV) es el principal agente etiológico entre las infecciones congénitas y la primera causa de sordera neurosensorial y daño cerebral en la infancia (1). EI CMV puede ser transmitido de la madre al feto en cualquier etapa de la gestación, cursando en forma asintomática para la madre en la mayoría de las veces, lo que dificulta determinar el momento en que se producirá la transmisión intrauterina del virus.

En los países desarrollados, la infección congénita por CMV se confirma en 0,5 a $1 \%$ de todos los recién nacidos vivos por cultivos al nacer (2). De éstos, solamente el $10 \%$ son sintomáticos y de ellos aproximadamente un $90 \%$ desarrollan secuelas neurológicas a largo plazo, como sordera neuro-sensorial (SNS), retardo mental (RM) y parálisis cerebral (PC). El 90\% de los recién nacidos CMV positivos son asintomáticos, pero no por ello libres de complicaciones, pues hasta el $23 \%$ de ellos desarrollan sordera y grados variables de retardo mental $(3,4)$.

El diagnóstico de la afección intrauterina severa se ha convertido en un desafío para la perinatología. Un subgrupo de casos de CMV congénito muere in útero y otro nace sintomático, con alta probabilidad de secuelas a largo plazo. La identificación in útero de estos pacientes invitaría a desarrollar terapias aplicables durante el embarazo. Para esto, se han buscado factores pronósticos, como la carga viral en líquido amniótico (5), alteraciones de pruebas hepáticas, coagulopatía fetal, etc. Hasta ahora, las aparición en el período fetal de ventriculomegalia, calcificaciones cerebrales o restricción de crecimiento son los signos de mayor valor pronóstico para nacer sintomático (6).

Además del tratamiento postnatal con ganciclovir (7) para disminuir la incidencia de sordera neurosensorial en niños sintomáticos, hay nuevas experiencias con valaciclovir y ganciclovir (8) oral en mujeres embarazadas. Una experiencia reciente con valaciclovir (9) oral mostró que $8 \mathrm{~g} /$ día permitía alcanzar concentraciones terapéuticas en sangre fetal. Esto abrió una posibilidad que debe aún ser demostrada tanto para la disminución de la tasa de transmisión vertical como para la disminución de las manifestaciones de una infección congénita constituida. Sin embargo, es necesario buscar nuevos marcadores de infección congénita que permitan seleccionar a la población potencialmente beneficiaria de tratamientos intrauterinos y evitar así las secuelas a largo plazo.

Históricamente las estrategias de screening de enfermedades infecciosas se han basado en la de- tección de una respuesta inmune. Desconcertantemente, un estudio publicado en 1999 (10) ha mostrado que en la enfermedad por CMV, la inmunidad materna preexistente no previene la infección fetal en el embarazo actual, a diferencia de lo que ocurre con la rubéola o la toxoplasmosis. Se ha demostrado transmisión vertical en casos de reinfección y de reactivación de la enfermedad, por lo que el feto de una paciente embarazada con seroconversión previa no estaría protegido. La razón es la infección por nuevos serotipos (11). La transmisión intrauterina en mujeres inmunes explica la relación directa entre seroprevalencia e incidencia de infección congénita para una población dada.

El objetivo de este estudio es conocer la seroprevalencia actual en la población obstétrica de un hospital público de Santiago, para definir si en el futuro es planteable un tamizaje universal para el diagnóstico precoz de infección por CMV en nuestras embarazadas y recién nacidos por estudios de seroconversión o de detección directa en los neonatos.

\section{MATERIAL Y MÉTODO}

Estudio prospectivo en 583 mujeres que ingresaron consecutivamente en trabajo de parto al Hospital Padre Hurtado durante los meses de mayo y junio del 2006. Se decidió este momento por ser una instancia en que había tiempo para que las pacientes pudieran decidir su participación, y para aprovechar el momento de la venopunción para realizar el examen de IgG anti CMV. No se incluyeron pacientes en que se realizó una cesárea electiva. El estudio fue aprobado por el Comité de Investigaciones del Servicio de Salud Sur Oriente.

Las pacientes recibieron una invitación a participar por escrito y firmaron un consentimiento informado. No se informó el resultado a las pacientes. En el preparto se obtuvo $5 \mathrm{ml}$ de sangre,que fue almacenada a $4^{\circ} \mathrm{C}$. Se realizó un test de ELISA para detectar IgG contra CMV en sangre materna (Zeus Scientific, New Jersey, EEUU).

El estudio contó con el financiamiento del programa de proyectos de investigación para especialistas en formación (Resident's Grant) de la Academia Americana de Pediatría.

\section{RESULTADOS}

Se obtuvo muestras de 583 mujeres en trabajo de parto obteniéndose 552 (95\%) casos positivos para IgG anti CMV, con $13(2 \%)$ negativos y 18 $(3 \%)$ indeterminados.

La mediana de la edad de las pacientes fue 24 
años (rango: 13 a 44 años). La paridad correspondió a 262 nulíparas (rango: 0 a 9 hijos previos). Las mujeres seronegativas tienen menor edad que las seropositivas sin ser estadísticamente significativo (Mann Whitney $p=0,26)$, pero tenían una menor paridad $(p=0,017)$. En la Figura 1 se presenta la edad promedio y 2 desviaciones estándar según el resultado del examen.

En la Tabla I mostramos el número de casos positivos según tramo de edad. Se encontró una correlación entre mayor grupo etario y mayor porcentaje de seropositivos (Spearman, $R=0,955$, $\mathrm{p}=0,019)$.

El número de indeterminados superó a la de negativos, ocurriendo en un $3 \%$ de las muestras. Esto es importante pues al momento de aplicar su uso

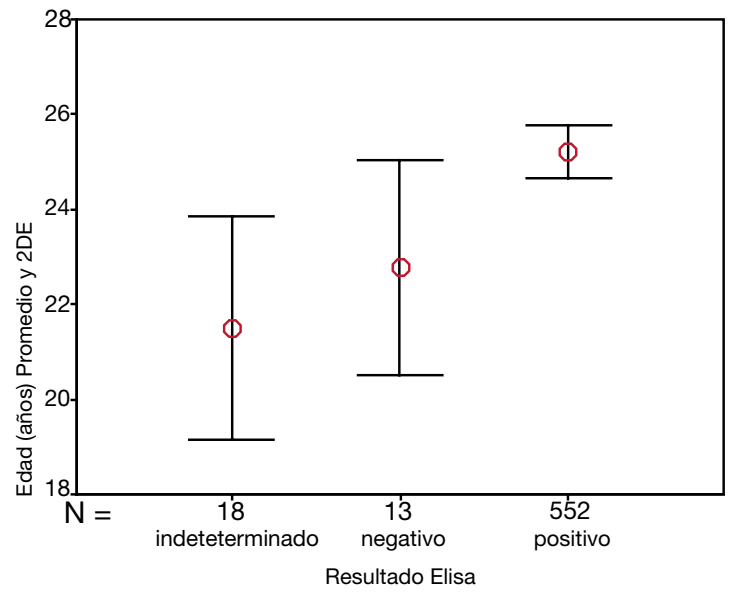

Figura 1. Edad promedio de pacientes IgG positivas, indeterminadas y negativas.

Tabla I

PORCENTAJE DE SEROPOSITIVIDAD POR RANGOS DE EDAD DE LAS MUJERES AL PARTO

\begin{tabular}{ccccc}
\hline Edad & $\mathrm{n}$ & positivos & negativos & positivos \\
\hline $10-14$ & 5 & 5 & 0 & $100,0 \%$ \\
$14-19$ & 138 & 130 & 2 & $94,2 \%$ \\
$20-24$ & 152 & 139 & 6 & $91,4 \%$ \\
$25-29$ & 150 & 142 & 5 & $94,7 \%$ \\
$30-34$ & 81 & 79 & 0 & $97,5 \%$ \\
$35-39$ & 41 & 41 & 0 & $100,0 \%$ \\
$40-44$ & 16 & 16 & 0 & $100,0 \%$ \\
\hline Total & 583 & 552 & 13 & $94,6 \%$
\end{tabular}

en grandes poblaciones puede generar un exceso de exámenes confirmatorios en estos pacientes. En este estudio no se realizaron exámenes confirmatorios.

El rango de la edad gestacional al parto fue 26 a 41 semanas. La edad gestacional no fue diferente entre seropositivas $(38,9 \pm 1,3$ semanas) y seronegativas (39,6 \pm 1,0 semanas) (Mann Whitney, $\mathrm{p}=0,54)$.

Ningún niño presentó CMV congénito sintomático al nacer.

\section{DISCUSIÓN}

La principal observación de nuestro estudio es la elevada seroprevalencia en nuestra población de embarazadas. Sin embargo, estudios prospectivos de mujeres embarazadas mostraron una tasa de adquisición de CMV para mujeres en edad fértil de $2 \%$ por año en el nivel socioeconómico medio-alto y de $6 \%$ anual en los niveles bajos (12). Esto no es concordante con la alta seroprevalencia, pues no habría una población seronegativa suficiente que pueda infectarse a estas tasas. Esto, sumado al hecho de que existe la reinfección, la serología en la embarazada no tendría una sensibilidad ni especificidad adecuada para el diagnóstico de infección congénita por CMV. La alta seropositividad desde edades maternas tempranas hace suponer que la gran mayoría de las infecciones congénitas se producirían en la reinfección.

La seropositividad para CMV estudiada en 1985 en Santiago, Chile, por Vial y cols (12), fue de $92 \%$ para una población de 833 gestantes de clase media y baja. El estudio incluyó a embarazadas con menos de 20 semanas de gestación controladas en la Universidad Católica y en el Servicio Sur Oriente de Santiago. La edad promedio y la edad gestacional promedio al momento de la obtención de sangre fue de 24,5 años y 18 semanas para los grupos de clase baja y 27,2 años y 14 semanas para los de clase media, respectivamente. Las pruebas fueron realizadas en la Universidad de Birmingham por los autores, estudiando también Herpes simplex, Rubéola, Hepatitis B y Toxoplasmosis. La prevalencia de IgG fue aumentando entre los 15 y 35 años de edad, de $70 \%$ a $90 \%$ en las mujeres de clase media y de $90 \%$ a cerca de $100 \%$ en las de clase baja, lo que es comparable con los resultados de nuestro estudio y demuestra que no ha cambiado en el tiempo.

Un estudio efectuado por Suárez y cols (13), buscó la ocurrencia de primoinfección en 1062 embarazadas, considerado en esa época como la principal forma de transmisión vertical de CMV, pues se 
estimaba que las reinfecciones eran infrequentes y de bajo riesgo de transmisión. Se encontró una prevalencia de $95 \%$ en 939 mujeres beneficiarias de consultorios del área norte de Santiago y $69 \%$ en 123 estudiantes de la Universidad de Chile. En este interesante estudio se siguió a las mujeres seronegativas, repitiendo el ELISA el segundo y tercer trimestre del embarazo. Hubo 47 y 37 embarazadas seronegativas en cada grupo. Dos de estas 84 presentaron seroconversión y sólo un recién nacido presentó un cultivo positivo para CMV. Por otra parte las poblaciones no eran similares pues la edad promedio (27años vs $23 a n ̃ o s, ~ p<0,01)$ y la paridad ( $70 \%$ multíparas vs $11,4 \%$ multiparas, $p<0,01$ ) eran mayores en la mujeres beneficiarias de los consultorios. El estudio de primoinfección realizado por Suárez y cols (13), mostró una incidencia de 1 caso de CMV congénito en 1000 embarazadas, lo que es mucho menor que la frecuencia de $1 \%$ de todos los recién nacidos vivos, reportada en países desarrollados. Esto puede deberse a que solo se estudió los recién nacidos con seroconversión materna, lo cual correspondió al $8 \%$ de la población de mujeres estudiadas. Esto hace pensar que existe un elevado subdiagnóstico.

El estudio más reciente fue publicado en 1997 por Abarca y cols (14), quienes estudiaron 560 personas sanas de ambos sexos, entre 6 meses y 30 años edad, no gestantes. La seroprevalencia fue de $60 \%$ en promedio, siendo de $39 \%, 64 \%$ y $75 \%$ en poblaciones de clase alta, media y baja, respectiva- mente. Este estudio también demostró una mayor seroprevalencia a mayor edad de la persona, sin diferencias entre los sexos.

IgM específico contra CMV se positiviza en 1 a 3 meses luego de la primoinfección (15) y puede persistir en niveles detectables por más de un año. También tiene el inconveniente de tener falsos positivos, como lo demuestra un estudio con 325 casos derivados por IgM positivos en que la repetición del estudio sólo confirmó la positividad en un 58\% de las pacientes (16).

Las experiencias chilenas se resumen en la Tabla II.

\section{CONCLUSIÓN}

Nuestra población tiene una alta seroprevalencia, y debido a la posibilidad de infección en mujeres seropositivas, se sospecha que la infección congénita por CMV puede ser frecuente. El screening para infección congénita con IgG no sería costo efectivo tanto por la baja posibilidad de detectar seroconversiones como por la posibilidad de reinfección. La alta prevalencia hace sospechar una alta frecuencia de la enfermedad y obliga a buscar el virus directamente en los fetos o recién nacidos, mediante detección de CMV en sangre, orina o saliva del recién nacido, ya sea por cultivo viral o PCR. Se requiere un estudio de prevalencia en recién nacidos por PCR o cultivos para poder cuantificar la real magnitud del problema.

Tabla II

PUBLICACIONES CHILENAS CON PREVALENCIA DE IGG ANTI CMV

\begin{tabular}{|c|c|c|c|c|c|}
\hline Autores & Año & $\begin{array}{c}\text { Nivel } \\
\text { socio- } \\
\text { económico }\end{array}$ & Instancia & $\mathrm{N}$ & Prevalencia \\
\hline \multirow{2}{*}{ Vial y cols $^{12}$} & \multirow{2}{*}{1985} & Bajo & Segundo & 456 & $96,5 \%$ \\
\hline & & Medio & trimestre & 321 & $86,8 \%$ \\
\hline \multirow{2}{*}{ Suarez y cols ${ }^{13}$} & \multirow{2}{*}{1994} & Bajo & Primer & 939 & $95 \%$ \\
\hline & & Alto & trimestre & 123 & $69,9 \%$ \\
\hline \multirow{3}{*}{ Abarca y cols ${ }^{14}$} & \multirow{3}{*}{1997} & Bajo & \multirow{3}{*}{$\begin{array}{l}\text { Niños y } \\
\text { adultos }\end{array}$} & 200 & $75,5 \%$ \\
\hline & & Medio & & 199 & $64,8 \%$ \\
\hline & & Alto & & 161 & $39,5 \%$ \\
\hline $\begin{array}{l}\text { Hospital Padre } \\
\text { Hurtado }\end{array}$ & 2005 & Bajo & $\begin{array}{l}\text { Trabajo } \\
\text { de parto }\end{array}$ & 583 & $94,6 \%$ \\
\hline
\end{tabular}




\section{BIBLIOGRAFÍA}

1. Stagno S. Cytomegalovirus infection: a pediatrician's perspective. Curr Probl Pediatr 1986;16:629-67.

2. Istas AS, Demmler GJ, Dobbins JG, Stewart JA. Surveillance for congenital cytomegalovirus disease: a report from the National Congenital Cytomegalovirus Disease Registry. Clin Infect Dis 1995;20:665-70.

3. Noyola DE, Demmler GJ, Nelson CT, et al. Early predictors of neurodevelopmental outcome in symptomatic congenital cytomegalovirus infection. J Pediatr 2001;138:325-31.

4. Boppana SB, Fowler KB, Vaid Y, et al. Neuroradiographic findings in the newborn period and long-term outcome in children with symptomatic congenital cytomegalovirus infection. Pediatrics 1997;99:409-14.

5. Revello MG, Zavattoni M, Baldanti F, et al. Diagnostic and prognostic value of human cytomegalovirus load and IgM antibody in blood of congenitally infected newborns. J Clin Virol 1999;14:57-66.

6. Yamamoto M, Jacquemard F, Picone $\mathrm{O}$, et al. Prenatal ultrasound findings in 93 cases of fetal infection with cytomegalovirus. Correlation with amniotic fluid viral load and outcome. Am J Obstet Gynecol 2005;191: S55.

7. Kimberlin DW, Lin CY, Sanchez PJ, et al. Effect of ganciclovir therapy on hearing in symptomatic congenital cytomegalovirus disease involving the central nervous system: a randomized, controlled trial. J Pediatr 2003;143:16-25.

8. Puliyanda DP, Silverman NS, Lehman D, et al. Successful use of oral ganciclovir for the treatment of intrauterine cytomegalovirus infection in a renal allograft recipient. Transpl Infect Dis 2005;7:71-4.
9. Jacquemard F, Yamamoto M, Picone O, et al. Cytomegalovirus intrauterine infection: pharmacokinetics of valaciclovir admnistration to the mother and changes in DNA viral load in amniotic fluid and fetal blood. Br J Obstet Gynaecol 2007;114:1113-21.

10. Boppana S, Fowler K, Britt W, Stagno S, Pass R. Symptomatic congenital cytomegalovirus infection in infants born to mothers with preexisting immunity to cytomegalovirus. Pediatrics 1999;104:55-60.

11. Boppana S, Rivera L, Fowler K, Mach M, Britt W. Intrauterine transmission of cytomegalovirus to infants of women with preconceptional immunity. N Engl J Med 2001;344:1366-71.

12. Vial P, Torres-Pereyra J, Stagno S, et al. Estudio serológico para citomegalovirus, herpes simples, rubéola, hepatitis B y toxoplasma gondii en dos poblaciones de gestantes en Santiago, Chile. Bol Of Sanit Panam 1985;99:528-38.

13. Suárez $M$, Briones $H$, Luchsinger $V$, et al. Primoinfección por citomegalovirus en embarazadas de diferente condición socioeconómica. Rev Med Chile 1994;122:1153-7.

14. Abarca K, Vial $P$, Zamorano J, et al. Seroprevalencia de citomegalovirus y Toxoplasma gondii en población sana menor de 30 años en Santiago de Chile. Rev Med Chile 1997;125: 531-8.

15. Revello M, Gerna G. Diagnosis and Management of Human Cytomegalovirus infection in the mother, fetus and newborn infant. Clin Microbiol Rev 2002;15: 680715.

16. Revello M, Gerna G. Diagnosis and implications of human cytomegalovirus infection in pregnancy. Fet Matern Med Rev 1999;11:117-34. 